\title{
Microfunctions at the Boundary and Mild Microfunctions
}

\author{
By \\ Pierre SCHAPIRA* and Giuseppe ZAMPIERI**
}

\begin{abstract}
Let $X$ be a real manifold, $\mathscr{F}$ an object of $D^{b}(X)$, the derived category of the category of bounded complexes of sheaves of abelian groups on $X$. The functor $\mu$ hom $(\cdot, \cdot)$, defined in [3], appears to be a useful tool especially in the theory of boundary value problems for partial differential equations. The aim of the present paper is to calculate the stalk of $R \Gamma_{z} \mu$ hom $\left(\boldsymbol{N}_{\mathscr{Q}}, \mathscr{F}\right)$, when $\Omega$ is a convex (up to diffeomorphism) and open subset of a closed submanifold $M$ of $X$, and $Z$ is a closed convex proper cone of $T^{*} X$. As an application we show how to recover in a short and functorial way, the theory of mild microfunctions by Kataoka [5].
\end{abstract}

\$1. Let $X$ be a real manifold of class $C^{\infty}, T^{*} X$ the cotangent bundle to $X, \pi: T^{*} X \rightarrow X$ the projection, $o r_{X}$ the orientation sheaf on $X$. If $M$ is a closed submanifold of $X$ one denotes by $T_{M}^{*} X$ the conormal bundle to $M$ in $X$ and by $o r_{M / X}$ the relative orientation sheaf. In particular one denotes by $T_{X}^{*} X$ the zero section of $T^{*} X$. One sets $\dot{T}^{*} X=T^{*} X \backslash T_{X}^{*} X, \dot{\pi}=\left.\pi\right|_{\dot{T}^{*} x}$. For two subsets $S$ and $V$ of $X$, one denotes by $C(S, V)$ the normal cone of $S$ along $V$ (cf. [2], [3]).

Let $D(X)$ denote the derived category of the category of complexes of sheaves of abelian groups on $X$, and let $D^{b}(X)$ be the full subcategory consisting of complexes with bounded cohomology. To $\mathscr{F} \in \mathrm{Ob}\left(D^{b}(X)\right)$ and $A \subset X$ locally closed, one associates the microlocalization of $\mathscr{F}$ along $A$ :

$$
\mu_{A}(\mathscr{F})=\mu \operatorname{hom}\left(\boldsymbol{Z}_{A}, \mathscr{F}\right)
$$

where $\boldsymbol{Z}_{A}$ is the sheaf which is zero in $X \backslash A$ and the constant sheaf with stalk $\boldsymbol{Z}$ on $A$, and where $\mu$ hom $(\cdot, \cdot)$ is the bifunctor defined

Communicated by M. Kashiwara, July 27, 1987.

* Université Paris-Nord, 93430 Villetaneuse, France.

** Dip. Mat., Università, Via Belzoni, 7, 35131 Padova, Italy. 
in [3]. This is an object of $D_{\text {co iic }}^{b}\left(T^{*} X\right)$, the full subcategory of $D^{b}(X)$ consisting of complexes whose cohomology objects are constant on the orbits of $\boldsymbol{R}^{+}$in $T^{*} X$. Moreover :

$$
\begin{aligned}
& \left.R \pi_{*} \mu_{A}(\mathscr{F}) \cong \mu_{A}(\mathscr{F})\right|_{T_{X}^{*} X} \cong R \Gamma_{A}(\mathscr{F}) . \\
& \operatorname{SS}\left(\mu_{A}(\mathscr{F})\right) \subset C\left(\operatorname{SS}(\mathscr{F}), \operatorname{SS}\left(Z_{A}\right)\right), \\
& \operatorname{supp}\left(\mu_{A}(\mathscr{F})\right) \subset \operatorname{SS}(\mathscr{F}) \cap \operatorname{SS}\left(Z_{A}\right),
\end{aligned}
$$

(where $\mathrm{SS}(\mathscr{F})$ is the microsupport of $\mathscr{F}$ as defined in [3]). Let $M$ be a real submanifold of $X$ with codimension $n$ and let $\Omega$ be an open $C^{\infty}$-convex subset of $M$ (i.e. : at any $x \in X$ there is a local chart in which $\Omega$ is convex). We first note that $\mathbb{Z}_{\Omega}$ is cohomologically constructible (cf. [3]) and that:

$$
R \mathscr{H}_{o m}\left(\boldsymbol{Z}_{\Omega}, \boldsymbol{Z}_{X}\right)=R \Gamma_{\Omega}\left(\boldsymbol{Z}_{X}\right) \cong \boldsymbol{Z}_{\bar{\Omega}} \otimes o r_{M / X}[-n] \text {. }
$$

Thus applying Proposition 5.6.3 of [3] we obtain:

$$
R \Gamma_{T_{X}^{*} X}\left(\mu_{\Omega}(\mathscr{F})\right) \cong \mathscr{F}_{\bar{\Omega}} \otimes \operatorname{or}_{M / X}[-n]\left(\cong R \pi_{1} \mu_{\Omega}(\mathscr{F})\right),
$$

which gives a distinguished triangle in $D^{b}(X)$ :

$$
\mathscr{F}_{\bar{\Omega}} \otimes o r_{M / X}[-n] \longrightarrow R \Gamma_{\Omega}(\mathscr{F}) \longrightarrow R \dot{\pi}_{*} \mu_{\Omega}(\mathscr{F}) \stackrel{+1}{\longrightarrow} \text {. }
$$

Recall that a conic subset of a vector bundle $E$ is called proper if it contains no line. If $Z$ is such a set we denote by $Z^{\circ}$ the polar (closed) cone in the dual vector bundle and we set $Z^{o a}=-Z^{\circ}$.

Theorem 1. 1. Let $Z$ be a closed convex proper cone of $T^{*} X$ containing $\bar{\Omega} \underset{X}{\times} T_{X}^{*} X$ and let $x \in X$. Then for a suitable open neighborhood $X^{\prime}$ of $x$ in $X$, we have:

$$
H_{Z}^{j}\left(T^{*} X^{\prime}: \mu_{\Omega}(\mathscr{F}) \otimes o r_{M / X}\right)=\underset{\mathrm{lim}}{\longrightarrow} H^{j-n}(U ; \mathscr{F})
$$

where $U$ ranges through the family of open subsets of $X^{\prime}$ such that $C\left(X^{\prime} \backslash U, \bar{\Omega}\right) \cap \operatorname{Int} Z^{o a}=\emptyset$.

Proof. We assume from the beginning that $X$ is a vector space, $M$ a vector subspace of $X, \Omega$ a convex subset of $M$; set $l=\operatorname{dim} X$. We recall that $\mu_{\Omega}(\mathscr{F})=\mu_{\Delta}\left(R \mathscr{H}_{\text {om }}\left(q_{2}^{-1} Z_{\Omega}, q \mathscr{F}\right)\right)$, where $\Delta$ is the diagonal of $X \times X, q_{i}: X \times X \rightarrow X,(i=1,2)$, is the $i^{\text {th }}$ projection, and where $X$ is identified to $\Delta$ and $T^{*} X$ to $T_{\Delta}^{*}(X \times X)$ by the first projection on $T^{*} X \times T^{*} X$. 
Using [3] Proposition 2.3.2, we have :

$$
\begin{aligned}
& H^{j}\left(R \Gamma_{Z}\left(T^{*} X, \mu_{\Omega}(\mathscr{F})\right)\right) \\
& \cong \underset{\mathrm{W}}{\longrightarrow} H^{j-l}\left(R \Gamma\left(W, R \mathscr{H}_{\mathrm{Com}}\left(q_{2}^{-1} \boldsymbol{Z}_{\Omega}, q_{1}^{1}(\mathscr{F})\right)\right)\right) \otimes o r_{X},
\end{aligned}
$$

for $W$ open subset of $X \times X$ with :

$$
C_{\Delta}(X \times X \backslash W) \cap \text { Int } Z^{o a}=\emptyset .
$$

We also have:

$$
\begin{aligned}
R \Gamma\left(W, R \mathscr{H}_{\text {om }}\left(q_{2}^{-1} \boldsymbol{Z}_{\Omega}, q_{1}^{1} \mathscr{F}\right)\right) & \cong R \operatorname{Hom}\left(\boldsymbol{Z}_{W}, R \mathscr{H} \mathscr{H}_{\text {om }}\left(q_{2}^{-1} \boldsymbol{Z}_{\Omega}, q_{1}^{1} \mathscr{F}\right)\right) \\
& \cong R \operatorname{Hom}\left(\boldsymbol{Z}_{W \cap(X \times \Omega)}, q_{1}^{1} \mathscr{F}\right) \\
& \cong R \operatorname{Hom}\left(R q_{11} Z_{W \cap(X \times \Omega)}, \mathscr{F}\right) .
\end{aligned}
$$

We claim that we can choose a fundamental system for the family of $W$ in (1.10) such that:

(1.11) $q_{1}^{-1}(x) \cap((X \times \Omega) \cap W)$ is convex for all $x \in X$.

In fact for a vector space $E$, and a linear subspace $M \subset E$, let us take an open cone $r \subset T_{M} E$ with convex fibers, and choose a linear projection $q: E \rightarrow M$. Then according to [1] Lemma 1, we can find a fundamental system of open sets $W \subset E$, with $C_{M}(E \backslash W) \cap \gamma=\emptyset$, such that $\left.q\right|_{W}$ has convex fibers. We apply the above remark with $E=X \times X, M=4, \gamma=$ Int $Z^{\circ a}, q=q_{1}$, and then get (1.11).

Now note that:

(1.12) $\left(R q_{1 !} \boldsymbol{Z}_{W \cap(X \times \Omega)}\right)_{x}=R \Gamma_{c}\left(q_{1}^{=1}(x) \cap(W \cap(X \times \Omega)) ;\left.\boldsymbol{Z}_{X \times X}\right|_{q_{1}^{-1}(x)}\right), x \in X$. If $x \notin q_{1}(W \cap(X \times \Omega))$, we find 0 in (1.12). Otherwise $q_{1}^{-1}(x) \cap(W \cap$ $(X \times \Omega))=W \cap(\{x\} \times \Omega)$ is a non-empty open subset of $M$. If we assume in addition that this is convex then the term in the right side of (1.12) is isomorphic to:

$$
R \Gamma_{c}\left(M ; Z_{M}\right)=Z[-\operatorname{dim} M]
$$

(for a choice of an orientation on $M$, i.e. an isomorphism $o r_{M} \cong \boldsymbol{Z}_{M}$ ). We have therefore proved that if (1.11) is fulfilled, then:

$$
R q_{1 !}\left(\boldsymbol{Z}_{W \cap(X \times \Omega)}\right)=\boldsymbol{Z}_{q_{1}(W \cap(X \times \Omega))} \otimes o r_{M}[-l+n] .
$$

We summarize up the results established until now by:

$$
\left\{\begin{array}{l}
H^{j}\left(R \Gamma_{z}\left(T^{*} X, \mu_{\Omega}(\mathscr{F})\right)\right) \\
\quad=\underset{W}{\lim } H^{j-n}\left(R \Gamma\left(q_{1}(W \cap(X \times \Omega)) ; \mathscr{F}\right)\right) \otimes o r_{M / X}, \\
\text { for } W \text { satisfying (1.10) and (1.11). }
\end{array}\right.
$$


We need now two lemmas.

Lenima 1.2. For any subsets $A \subset X \times X$ and $B \subset X$, we have:

$$
C_{\Delta}(X \times X \backslash A) \supset C\left(X \backslash q_{1}((X \times B) \cap A), \bar{B}\right),
$$

(under the identification $T^{*} X \cong T_{\Delta}^{*}(X \times X)$ by the first projection defined on $\left.T^{*} X \times T^{*} X\right)$.

Proof. Let $\theta \in C\left(X \backslash q_{1}((X \times B) \cap A), \bar{B}\right)$. There exist sequences $\left\{\left(x_{n}\right.\right.$, $\left.\left.y_{n}\right)\right\}_{n} \subset X \times X$, and $\left\{c_{n}\right\}_{n} \subset \boldsymbol{R}^{+}$such that:

$$
\begin{aligned}
& \left(x_{n}, y_{n}\right) \underset{n}{\longrightarrow}(x, x), c_{n}\left(x_{n}-y_{n}\right) \longrightarrow{ }_{n} \\
& y_{n} \in \bar{B}, \quad\left(x_{n}, z\right) \notin A \quad \forall z \in B .
\end{aligned}
$$

We choose a sequence $z_{n} \underset{n}{\longrightarrow} x, z_{n} \in B$ such that:

$$
c_{n}\left(z_{n}-y_{n}\right) \underset{n}{\longrightarrow} 0 \text {. }
$$

It then follows :

$$
c_{n}\left(\left(x_{n}, z_{n}\right)-\left(y_{n}, y_{n}\right)\right) \underset{n}{\longrightarrow}(\theta, 0) .
$$

Hence $\theta \in C_{\Delta}(X \times X \backslash A)$.

Lemma 1.3. For any open set $U \subset X$ and any set $B \subset X$, there exists an open set $W \subset X \times X$ such that:

$$
\begin{aligned}
& q_{1}((X \times B) \cap W) \subset U . \\
& C_{\Delta}(X \times X \backslash W) \subset C(X \backslash U, \bar{B}) .
\end{aligned}
$$

Proof. Let us set:

$$
W=(U \times X) \cup(X \times(X \backslash \bar{B})) .
$$

Then (1.16) is clear.

On the other hand let us be given three sequences:

$$
\begin{array}{ll}
\left\{\left(x_{n}, y_{n}\right)\right\}_{n} \subset X \times X \backslash W, & \left(x_{n}, y_{n}\right) \underset{n}{\longrightarrow}(x, x), \\
\left\{\left(z_{n}, z_{n}\right)\right\}_{n} \subset \Delta, & \left(z_{n}, z_{n}\right) \underset{n}{\longrightarrow}(x, x), \\
\left\{c_{n}\right\}_{n} \subset \boldsymbol{R}^{+}, &
\end{array}
$$

such that:

$$
c_{n}\left(\left(x_{n}, y_{n}\right)-\left(z_{n}, z_{n}\right)\right) \underset{n}{\longrightarrow}\left(\theta, \theta^{\prime}\right)
$$

It follows that: 


$$
c_{n}\left(x_{n}-y_{n}\right) \underset{n}{\longrightarrow} \theta-\theta^{\prime}
$$

with $y_{n} \in \bar{B}$ and $x_{n} \notin U$ due to $\left(x_{n}, y_{n}\right) \notin W$.

It follows :

$$
\theta-\theta^{\prime} \in C(X \backslash U, \bar{B}),
$$

which concludes the proof of (1.17).

End of proof of Theorem 1.1. Let:

$$
\mathscr{U}=\left\{U \subset X ; U \text { is open and } C(X \backslash U, \bar{\Omega}) \cap \operatorname{Int} Z^{\circ a}=\phi\right),
$$

and :

$\mathscr{U}^{\prime}=\left\{U=q_{1}(W \cap(X \times \Omega)) ; W \subset X \times X\right.$ is open and $C_{\Delta}(X \times X \backslash W) \cap$ Int $\left.Z^{\circ a}=\emptyset\right\}$.

Choose $A=W, B=\Omega$, in Lemmas 1.2 and 1.3. Then by the first lemma we have $\mathscr{U}^{\prime} \subset \mathscr{U}$ and by the second we obtain that for any $U \in \mathscr{U}$ there exists $U^{\prime} \in \mathscr{U}^{\prime}$ such that $U^{\prime} \subset U$. The proof is complete.

\$2. Let $M$ be a real analytic manifold of dimension $n, X$ a complexification of $M, \Omega \subset M$ an open subset with analytic boundary $N=\partial \Omega(\Omega$ on one side of $N), Y$ a complexification of $N$ in $X$. For any locally closed set $A \subset X$, (in particular for $A=\Omega$ ), we set:

$$
\mathscr{C}_{A \mid X}=\mu_{A}\left(\mathcal{O}_{X}\right) \otimes o r_{M i X}[n],
$$

$\mathcal{O}_{X}$ being the sheaf of holomorphic function on $X$.

Proposition 2.1. The complex $\left(\mathscr{C}_{\Omega \mid X}\right)_{\dot{T}_{M}^{*} X}$ is concentrated in degree 0 and when identifying it to its 0-th cohomology object, it is a conically flabby sheaf (i.e. its image in $T_{M}^{*} X / \boldsymbol{R}^{+}$is a flabby sheaf).

Proof. The first part of the statement follows from [8. II] Proposition 3.1. As for the second one, consider the distinguished triangle:

$$
\mathscr{C}_{M \backslash \Omega \mid X} \longrightarrow \mathscr{C}_{M \mid X} \longrightarrow \mathscr{C}_{\Omega \mid X} \stackrel{+1}{\longrightarrow} \text {. }
$$

On account of the well known flabbiness of $\mathscr{C}_{M \mid X^{\prime}}$ it is enough to prove that: 


$$
\left\{\begin{array}{l}
H^{i}\left(U ;\left.\mathscr{C}_{M \backslash \Omega \mid X}\right|_{\substack{N \times T_{M}^{* X} \\
\text { X }}}\right)=0, \text { for any } i \geq 1 \text { and for any conic open set } \\
U \subset N \times \dot{T}_{M}^{*} X .
\end{array}\right.
$$

On the other hand using the exact sequence :

$$
\left.\left.\left.0 \longrightarrow \mathscr{C}_{N \mid X}\right|_{N \times T_{M}^{*} X} \longrightarrow\left(\mathscr{C}_{M \backslash \Omega \mid X} \oplus \mathscr{C}_{\bar{\Omega} \mid X}\right)\right|_{N \times T_{M}^{*}} \longrightarrow \mathscr{C}_{M \mid X}\right|_{N \times T_{M}^{*}} \longrightarrow 0,
$$

it is enough to prove that:

(2.2) $H^{i}\left(U ; \mathscr{C}_{N \mid X}\right)=0$ for any $i \geq 1$, and for any open cone $U \subset \dot{T}_{N}^{*} X$.

We also note that since flabbiness is a local property, then it is enough to prove that for any $x \in N,(2.2)$ is fulfilled with $U \subset$ $\pi^{-1}(B)$, for a suitable neighborhood $B$ of $x$. We then write in local coordinates in $B$ :

$$
M=\boldsymbol{R} \times N, X=\boldsymbol{C} \times Y, \tilde{M}=\boldsymbol{C}^{\boldsymbol{R}} \times N, \tilde{X}=\boldsymbol{C} \times \overline{\boldsymbol{C}} \times Y,
$$

where $C^{R}$ is the real underlying manifold to $C$, and $C \times \bar{C}$ its complexification by the diagonal embedding $C \longleftrightarrow C \times \bar{C}$.

We may also identify $T_{N}^{*} X$ and $T_{\tilde{M}}^{*} \tilde{X} \cap\left((\mathbb{C} \times\{0\} \times Y) \underset{X}{\times} T^{*} \tilde{X}\right)$, and we have an exact sequence on $T_{\tilde{M}}^{*} \tilde{X}$ (cf. [2] Theorem 6.1.2):

$$
0 \longrightarrow \mathscr{C}_{N \mid X} \longrightarrow \mathscr{C}_{\tilde{M} \mid \tilde{X}} \underset{\bar{z}_{1}}{\longrightarrow} \mathscr{C}_{\tilde{M} \mid \tilde{X}} \longrightarrow 0,
$$

where $z_{1}$ is a holomorphic coordinate on $C$.

Since $\bar{z}_{1}: \Gamma\left(U ; \mathscr{C}_{\tilde{M} \mid \tilde{X}}\right) \rightarrow \Gamma\left(U ; \mathscr{C}_{\tilde{M} \mid \tilde{X}}\right)$ is surjective for any open conic set $U \subset \dot{T}_{\tilde{M}}^{*} \tilde{X}$, we then get (2.2) (with $U \subset \dot{\pi}^{-1}(B)$ ) which completes the proof.

(For vanishing theorems as (2.2), we also refer to [6]).

Recall that one has an isomorphism:

$$
\Gamma_{\Omega}\left(\mathscr{B}_{M}\right) \underset{\alpha}{\sim} \pi_{*}\left(\mathscr{C}_{\Omega \mid X}\right)_{T_{M}^{*}} \cdot
$$

$\mathscr{B}_{M}$ being the sheaf of Sato's hyperfunctions.

Following [8] we then define the microsupport at the boundary $\mathrm{SS}_{\Omega}$ by :

$$
\mathrm{SS}_{\Omega}(f)=\operatorname{supp}(\alpha(f)), \quad f \in \Gamma\left(\Omega ; \mathscr{B}_{M}\right) .
$$

On account of Proposition 2.1, we immediately obtain: 
Corollary 2.2. Let $Z=\operatorname{SS}_{\Omega}(f)$ and let $Z, \subset T_{M}^{*} X, j=1, \ldots, N$, be closed cones with $\cup Z,=Z$. Then we can decompose:

$$
f=\sum_{\jmath} f_{,} \text {, with } f_{J} \text { in } \Gamma\left(\Omega ; B_{M}\right) \text { and } \operatorname{SS}_{\Omega}\left(f_{J}\right) \subset Z_{\jmath} \cup\left(M \underset{X}{\times} T_{X}^{*} X\right) \text {. }
$$

Let us denote by:

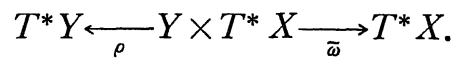

the canonical maps associated to $Y \longleftrightarrow X$.

Definition 2.3. We set:

$$
\begin{aligned}
& \hat{\mathscr{C}}_{\Omega \mid N}=R \rho_{\mid} \widetilde{\varpi}^{-1} \mathscr{C}_{\Omega \mid X}, \\
& \hat{\mathscr{B}}_{\Omega \mid N}=\left.\hat{\mathscr{C}}_{\Omega \mid N}\right|_{T_{Y}^{*} Y^{*}}
\end{aligned}
$$

We shall see that $\hat{\mathscr{C}}_{\Omega \mid N}$ (resp. $\hat{\mathscr{B}}_{\Omega \mid N}$ ) is concentrated in degree zero and coincides with the sheaf of Kataoka's mild microfunctions (resp. hyperfunctions), defined in [5]. That is why we have used the same notations as Kataoka's.

In the sequel, we shall identify an object $\mathscr{F}$ of $D^{b}(X)$ concentrated in degree zero and the sheaf $H^{0}(\mathscr{F})$.

Proposition 2.4. (i) The complex $\hat{\mathscr{C}}_{\Omega \mid N}$ is concentrated in degree zero.

(ii) The sheaf $\left(\hat{\mathscr{C}}_{\Omega \mid N}\right)_{\dot{T}_{N}^{*} Y}$ is conically soft.

(iii) There is a natural exact sequence of sheaves on $N$ :

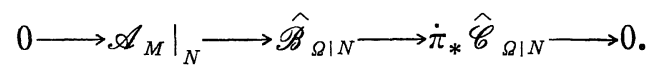

Proof. Set $L=\operatorname{SS}\left(\boldsymbol{Z}_{\Omega}\right) \backslash T_{M}^{*} X$. Then $\left(\mathscr{C}_{\Omega \mid X}\right)_{L}$ is isomorphic to $\left(\mathscr{C}_{N \mid X}\right)_{L}[1]$. Consider the distinguished triangle:

$$
\left(\mathscr{C}_{N \mid X}\right)_{L}[1] \longrightarrow \mathscr{C}_{\Omega \mid X} \longrightarrow\left(\mathscr{C}_{\Omega \mid X}\right)_{T_{M}^{*} X} \vec{\longrightarrow}
$$

and apply the functor $R \rho_{!} \varpi^{-1}$ to it.

Set $\mathscr{F}=R \rho_{!} \varpi^{-1}\left(\left(\mathscr{C}_{N \mid X}\right)_{L}[1]\right)$ and $\mathscr{G}=R \rho_{!} \varpi^{-1}\left(\left(\mathscr{C}_{\Omega_{\mid X} X}\right)_{T_{M}^{*}}\right)$.

By Proposition 2. 1, the complex $\mathscr{G}_{\dot{T}_{N}^{*}{ }^{*}}$ is concentrated in degree zero and is conically soft. On the other hand, the sheaf $\mathscr{C}_{N \mid X}$ satisfies the principle of analytic continuation along the leaves of $Y \underset{X}{X} T^{*} X$. This implies $\rho_{!} \varpi^{-1} \mathscr{C}_{N \mid X}=0$ and the complex $\mathscr{F}$ is concentrated in 
degree $\geq 0$. Consider the distinguished triangle deduced from (2.3):

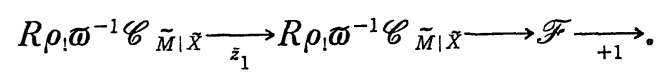

The sheaf $\mathscr{C}_{\tilde{M} \mid \tilde{X}}$ being conically flabby on $\dot{T}_{M}^{*} X$, we get that $\mathscr{F}$ is concentrated in degree zero and is conically soft on $\dot{T}_{N}^{*} Y$. Now consider the distinguished triangle:

$$
\left.R \pi_{Y !} R \rho_{!} \varpi^{-1} \mathscr{C}_{\Omega \mid X} \longrightarrow\left(R \rho_{!} \varpi^{-1} \mathscr{C}_{\Omega \mid X}\right)\right|_{T_{Y}^{*} Y} \longrightarrow R \dot{\pi}_{*} R \rho_{!} \varpi^{-1} \mathscr{C}_{\Omega \mid X} \underset{+1}{\longrightarrow} .
$$

By (1.7) the first term is nothing but $\left.\mathscr{A}_{M}\right|_{N}$. Moreover $\left.\hat{\mathscr{C}}_{\Omega \mid X}\right|_{\dot{T}_{N}^{*} Y}$ being conically soft, $R \dot{\pi}_{*} \hat{\mathscr{C}}_{\Omega \mid X}=\dot{\pi}_{*} \hat{\mathscr{C}}_{\Omega \mid X}$. This completes the proof.

The main property of the sheaf $\hat{\mathscr{C}}_{g \mid N}$ is that there exists a boundary value morphism in $\mathscr{C}_{N \mid Y}$. In fact recall ([8]) that the morphism $\boldsymbol{Z}_{\bar{\Omega}} \rightarrow \boldsymbol{Z}_{N}$ defines the morphism $\boldsymbol{Z}_{N} \rightarrow \boldsymbol{Z}_{\Omega} \otimes$ or $_{N / M}[-1]$ thus the morphism :

$$
\mathscr{C}_{\Omega \mid X} \longrightarrow \mathscr{C}_{N \mid X} \otimes o r_{N / M}[1] .
$$

By Proposition 2.3.5 of [3] we also have a morphism:

$$
R \rho_{1} \varpi^{-1} \mathscr{C}_{N \mid X}[1] \longrightarrow \mathscr{C}_{N \mid Y} \otimes o r_{N / M} .
$$

Combining (2.8) and (2.9) we get:

Proposition 2.5. There exists a natural "boundary value" morphism:

$$
b: \quad \hat{\mathscr{C}}_{\Omega \mid N} \longrightarrow \mathscr{C}_{N \mid Y}
$$

which induces a morphism:

$$
\text { b: } \quad \hat{\mathscr{B}}_{\Omega \mid N} \longrightarrow \mathscr{B}_{N} .
$$

Remark that the natural morphism $R \rho_{!} \widetilde{\Phi}^{-1} \mathscr{C}_{M \mid X} \rightarrow \mathscr{C}_{N \mid Y}$ (cf. [7]) factorizes through the morphism $\hat{\mathscr{C}}_{\Omega \mid N} \rightarrow \mathscr{C}_{N \mid Y}$, via the natural morphism $\mathscr{C}_{M} \rightarrow \mathscr{C}_{\Omega \mid X}$, induced by $\boldsymbol{Z}_{\Omega} \rightarrow \boldsymbol{Z}_{M}$. Similarly, the restriction morphism $\left.\mathscr{A}_{M}\right|_{N} \rightarrow \mathscr{A}_{N}$ is induced by $\hat{\mathscr{B}}_{\Omega \mid N} \rightarrow \mathscr{B}_{N}$.

As an application of Theorem 1.1, we get:

Proposition 2.6. Let $Z \subset T_{N}^{*} Y$ be a closed convex proper cone containing the zero-section $N \underset{Y}{\underset{Y}{X}} T_{Y}^{*} Y$. Let $x \in N$. Then for any $j \in Z$ : 


$$
H^{j}\left(R \Gamma_{z} \mathscr{C}_{\Omega \mid N}\right)_{x}=\underset{U}{\lim } H^{j}\left(U ; \mathcal{O}_{X}\right)
$$

where $U$ ranges through the family of open subsets of $X$ which satisfy the following property:

there exists a closed convex proper cone $\tilde{Z} \subset T^{*} X$ such that $\tilde{Z}$ contains the zero-section $M \underset{X}{\times} T_{X}^{*} X, \rho$ is proper on $\widetilde{\sigma}^{-1}(\tilde{Z}), \rho \widetilde{\varpi}^{-1}(\tilde{Z})=Z$, and there exists an open neighborhood $X^{\prime}$ of $x$ in $X$ with:

$$
C\left(X^{\prime} \backslash U, \bar{\Omega}\right) \cap \operatorname{Int} \tilde{Z}^{o a}=\emptyset .
$$

Remark 2.7. The sheaf $\hat{\mathscr{C}}_{\Omega \mid N}$ coincides with the sheaf of Kataoka's mild microfunctions [5]. To check it, it is enough to restrict ourselves to $\dot{T}_{N}^{*} Y$, in view of the exact sequence (2.7). Then both sheaves are conically soft, and it is enough to verify that they have same sections supported by closed convex proper cones, but this follows from Proposition 2.6.

\section{References}

[1] Bros, J. and Iagolnitzer, D., Tuboïdes dans $\boldsymbol{C}^{n}$ et généralisation d'un théorème de Cartan et Grauert. Ann. Inst. Fourier (Grenoble), 26 (1976), 49-72.

[2] Kashiwara, M. and Schapira, P., Microhyperbolic systems. Acta Math., 142 (1979), $1-55$.

[3] Microlocal study of sheaves, Astérisque, 128 (1985).

[4] Kataoka, K., On the theory of Radon transformations of hyperfunctions. J. Fac. Sci. Univ. Tokyo Sect. IA Math., 28 (1981), 331-413.

[5] Microlocal theory of boundary value problem I and II, J. Fac. Sci. Univ. Tokyo Sect. IA Math., 27 (1980), 355-399, and 28 (1981), 31-56.

[6] Kataoka, K. and Tose, N., Vanishing theorem for the sheaf of microfunctions with holomorphic parameters. Flabbiness of the sheaf of 2-microfunctions, to appear.

[7] Sato, M., Kashiwara, M. and Kawai, T., Hyperfunctions and pseudodifferential equations, Lecture Notes in Math., Springer Verlag, 287 (1973), 265-529.

[8] Schapira, p., Front d'onde analytique au bord I et II. C. R. Acad. Sci., 302, n 10 (1986), et Sém. E. D. P. Ecole Polytechnique Exp. 13 (1986).

[9] Microfunctions for boundary value problems, Prospect in Algebraic Analysis. to appear. 
